\title{
Embolization of Vascular Tumors of the Head and Neck: A Review of Literature
}

\author{
Ankit Singla ${ }^{1}$, Nishant Singh ${ }^{2}$, Shivendra Singh ${ }^{3}$, Meghna Kumar ${ }^{4}$, Rahul Srivastava ${ }^{5}$ \\ ${ }^{1}$ Post Graduate Student, Department of Oral \& Maxillofacial Surgery, Rama Dental College-Hospital \& Research Centre, \\ ${ }^{2}$ Professor, Department of Oral \& Maxillofacial Surgery, Rama Dental College-Hospital \& Research Centre, ${ }^{3}$ Assistant Professor, \\ Department of Oral \& Maxillofacial Surgery, Rama Dental College-Hospital \& Research Centre, ${ }^{4}$ Post Graduate Student, \\ Department of Oral \& Maxillofacial Surgery, Rama Dental College-Hospital \& Research Centre, ${ }^{5}$ Professor, Department of \\ Oral Medicine and Radiology, Rama Dental College-Hospital \& Research Centre, India
}

Corresponding author: Dr. Shivendra Singh, Assistant Professor, Department of Oral \& Maxillofacial Surgery, Rama Dental College-Hospital \& Research Centre, India

DOI: http://dx.doi.org/10.21276/ijcmsr.2020.5.1.11

(c) BY-NC-ND

How to cite this article: Ankit Singla, Nishant Singh, Shivendra Singh, Meghna Kumar, Rahul Srivastava. Embolization of vascular tumors of the head and neck: a review of literature. International Journal of Contemporary Medicine Surgery and Radiology. 2020;5(1):A47-A51.

\section{A B S T R A C T}

Embolization of vascular tumors of the head and neck is an important adjunctive therapy to the surgical treatment of these tumors. Palliative embolization is the sole treatment in high risk patients. Preoperative embolization reduces intraoperative bleeding and shortens surgery time thereby decreasing morbidity and mortality. Study of the tumor vascular anatomy and adhering to principles can prove this approach to be safe and effective. Various embolic materials are available and can be safely applied for a given tumor and its vascular supply. This article aims to summarize the current methods and agents used in the embolization of head and neck vascular tumors.

Keywords: Embolization, Head And Neck Tumors, Embolic Agents

\section{INTRODUCTION}

Vascular anomalies are divided into two categories: tumors and malformations. These clinical entities are extremely different from one another but are often confused.A thorough medical history and the clinical examination is necessary to define any vascular anomaly. There are many different vascular tumors but Infantile hemangiomas are the most common. In 1982 Mulliken and Glowacki classified them on the basis of histology, biology, and clinical presentation. ${ }^{1}$ They divided vascular anomalies into two major categories: tumors and malformtions. ${ }^{2}$ In 1996, the International Society for the Study of Vascular Anomalies (ISSVA) adopted and modified Mulliken and Glowacki's original classification. ${ }^{2}$ This classification is now widely aacepted for the diagnosis and management of the vascular anomalies.

\section{Functional vascular anatomy}

An understanding of the anatomy of the external carotid artery (ECA) is essential for performing safe and effective embolization of vascular tumors of the head and neck because of the many anatomic variations, territorial anastomoses, and collateral supplies found in this region. ${ }^{3}$ The blood supply to the tumors of head and neck is derived from vasculature of the adjoining region and by branches of the External carotid artery (ECA) along with the vertebral artery (VA), internal carotid artery (ICA) and the thyrocervical or costocervical trunk.
Prevention of any serious complication requires knowledge and recognition of the territorial anastomosis. There are anastomotic pathways between the ECA, ICA, VA, ophthalmic artery, ascending cervical artery, deep cervical artery and spinal arteries which are embryological remnants. The most common dangerous anastomosis involve communications of the first- or second-order branches of the ECA i.e. the ascending pharyngeal (APA), occipital (OA), middle meningeal (MMA), accessory meningeal (AMA), and internal maxillary arteries (IMA) with the ICA or VA. Furthermore, MMA, IMA, superficial temporal and facial arteries can all anastomose with the ophthalmic artery. ${ }^{4}$ Surgeons need to be familiar with these connections when embolizing tumors within any of these vascular territories to avoid inadvertent passage of the embolic material to the retina or central nervous system. The ECA also supplies collateral circulation for the ICA and primary blood supply to the cranial nerves which can result in palsies of cranial nerves V, VII, IX, X, XI, and XII due to inappropriate embolization of the feeding branches to the vasa nervosum. So, selection of the correct embolic agent and if required, angiography of the vascular territory before embolization can help avoid damage to these structures.

\section{PRINCIPLES OF EMBOLIZATION}

Tumor embolization occludes the feeding vessels of the tumor due to which it can be used adjunctively with surgical 
resection or as a palliative measure. Treatment begins with first obtaining a cerebral angiogram so as to avoid unintended vessel occlusion. Pre-operative tumor embolization results in improved surgical outcome by reducing intraoperative blood loss and facilitating tumor resection. Indications for tumor embolization are: (1) to control surgically inaccessible arterial feeders, (2) to decrease surgical morbidity by reducing blood flow, (3) to shorten the operative procedure time, (4) to increase the chances of complete surgical resection, (5) to decrease the risk of damage to adjacent normal tissue, (6) to relieve intractable pain, (7) to decrease expected tumor recurrence, and (8) to allow better visualization of the surgical field with decreased overall surgical complication.

The embolization is ideally performed 24 to 72 hours before the surgical resection to allow time for maximum thrombosis of the occluded vessels and prevent recanalization of the occluded vessels. Ideal tumor embolization is achieved with the occlusion of very small vessels within a tumor, while sparing the supply to normal adjacent tissue. ${ }^{5}$ Liquid and particulate embolic materials are effective in penetrating small vessels. The procedure commonly begins with smaller sized embolic material to target small vessels and gradually increasing the particle size in successive injections.

The treatment begins with first obtaining a detailed cerebral angiogram that includes selective injections of the common carotid artery, ICA, ECA, VA, and the thyrocervical and costocervical trunks of the subclavian artery. ${ }^{6}$ If blood supply to the cranial nerves is suspected, provocative testing can be performed by injecting lidocaine and then observing for neurologic deficit. If a deficit is elicited then the microcatheter can be repositioned. ${ }^{7}$ Embolization approaches include transarterial, direct puncture and a combination of these both methods. Ideal tumor embolization is achieved with occlusion of the very small vessels within a tumor, while sparing supply to normal adjacent tissue. Liquid and particulate embolic materials are effective in penetrating small vessels, and meticulous use of these materials is essential in preventing unintended occlusions. Common practice is to begin with smaller sized embolic material to target small distal vessels and gradually increase particle size in successive injections of embolic material.

\section{EMBOLIZATION MATERIALS}

The goal of tumor embolization is to selectively occlude the ECA feeders using intratumoral deposition of the embolic material. ${ }^{7}$ The embolic agents commonly used include the following:

- polyvinyl alcohol

- trisacryl microspheres

- liquid n-butyl cyanoacrylate (n-BCA)

- ethyl-vinyl alcohol copolymer (EVOH) Onyx

- gelfoam pledgets

- microcoils

The embolic materials are basically classified into three different categories i.e. Particles, Liquid agents and Coils. The unique characteristics of each embolic material offer

\begin{tabular}{|l|l|}
\hline Vascular tumours & Vascular malformations \\
\hline Infantile hemangioma & $\begin{array}{c}\text { Slow flow: } \\
\text { Capillary malformation } \\
\text { Venous malformation } \\
\text { Lymphatic malformation }\end{array}$ \\
\hline $\begin{array}{l}\text { Congenital hemangioma } \\
\text { Rapidly involuting congenital hemangioma } \\
\text { Non-involuting congenital hemangioma }\end{array}$ & $\begin{array}{c}\text { Fast flow: } \\
\text { Arterial malformation } \\
\text { Arteriovenous malformation }\end{array}$ \\
\hline Tufted Angioma & Combined vascular malformation \\
\hline Kaposiform hemangioendothelioma & \\
\hline Spindle cell hemangioendothelioma & \\
\hline Epithelioid hemangioendothelioma & \\
\hline Other rare hemangioendotheliomas e.g. Composite, retiform & \\
\hline Dermatologic acquired e.g. Lobular capillary hemangioma & \\
\hline Angiosarcoma & \\
\hline \multicolumn{1}{|c|}{ Table-1: ISSVA classification of vascular anomalies } & \\
\hline
\end{tabular}

\begin{tabular}{|l|l|l|}
\hline Artery & Cranial nerve at risk & Notable anastomosis \\
\hline Superior Thyroid & X, Superior laryngeal nerve branch & Contralateral superior thyroid and inferior thyroid arteries \\
\hline Lingual & XII, extra cranial segment & Contralateral lingual artery, superior thyroid artery \\
\hline Facial & Rare direct CN association & Lingual artery, ophthalmic artery, rarely with ascending pharyngeal \\
\hline Ascending pharyngeal & IV,V, VI, VII, VIII, IX & $\begin{array}{l}\text { ICA, VA meningo-hypophyseal trunk, middle meningeal artery, } \\
\text { inferior tympanic artery }\end{array}$ \\
\hline Posterior auricular & VII & Meningeal anastomosis with MMA and ascending pharyngeal \\
\hline Occipital & VII & Trans-osseous to posterior fossa, muscular branches to VA \\
\hline Internal maxillary & III, V, VI & ICA, Ophthalmic artery \\
\hline Middle meningeal & V. VI. VII & Recurrent ophthalmic to eye and ICA, ILT \\
\hline Superficial Temporal & Rarely via anastomosis to VII and VIII & Ophthalmic artery via the supraorbital branch \\
\hline & Table-2: External carotid arteries, associated cranial nerves and notable anastomoses \\
\hline
\end{tabular}




\begin{tabular}{|l|l|l|}
\hline Embolic Agent & Advantages & \\
\hline & Particles & \\
\hline PVA & Microvascular penetration, Easy to use & Radiolucent, Irregular shapes may allow recanalization \\
\hline Microspheres & Microvascular penetration, Minimal clumping & Fragile, Many are radiolucent \\
\hline Gelfoam & $\begin{array}{l}\text { Medium to large vessel occlusions, Inexpensive, Easy } \\
\text { to use }\end{array}$ & Temporary \\
\hline n-BCA & Liquid agents & Rapid solidification and occlusion, Can flow into com- \\
plex angioarchitecture & $\begin{array}{l}\text { Slower solidification, can flow into complex angioar- } \\
\text { chitecture }\end{array}$ & Catheter retainment \\
\hline & Coils & Possible dislodgement and embolization \\
\hline Coils & Precise deployment, Useful in high-flow vessels & \multicolumn{2}{|c|}{ Table-3: Types of Embolization Materials } \\
\hline
\end{tabular}

several options for tumor devascularization (Table 3).

\section{PARTICLES}

\section{Polyvinyl alcohol}

Poly vinyl alcohol particles are embolic agents available in various sizes ranging from $45 \mu \mathrm{m}$ to over $1000 \mu \mathrm{m}$. Use of PVA particles of $45-150 \mu \mathrm{m}$ followed by $150-250 \mu \mathrm{m}$ can be successful in tumor embolization. Smaller particles $45-$ $150 \mu \mathrm{m}$ penetrate the capillary vascular bed of the tumor and aid in devascularization. These particles often devascularize to the extent that the tumor undergoes necrosis. Larger particles 150-250 $\mu \mathrm{m}$ embolize smaller arterioles in the tumor bed. If a dangerous anastomosis is suspected, larger particle sizes can be utilized to prevent accidental embolization of the anastomosing branches. Good blood flow should be ensured during embolization as anterograde flow in the supplying branches will carry the embolizing material to the target vasculature. Swift sequential injections with minimal delay in syringe changes are important to provide a near continuous and steady administration of particles because occlusion relies partly on particle aggregation. Flow arrest due to vasospasm or any other etiology may lead to reflux and increased risk of embolization of an unintended target. Use of small particles should be limited to the tumor bed as it may cause necrosis of the overlying skin in cases of extra-cranial tumors. Embolization should be continued until tumor blush disappears or there is prolonged contrast stagnation with increased resistance to further embolization. Tumors deriving blood supply from multiple feeders will require embolization through each of the supplying pedicles.

\section{Microspheres}

Microsphere embolics include trisacryl gelatin microspheres (Embospheres) and a newer hydrogel microsphere with a polymer coating (Embozene). These agents are nonabsorbable, round, embolic agents with precise size. These agents differ from PVA particles due to uniform size and shape, which theoretically improves distal penetration. The hydrophilic coating of the Embosphere is intended to reduce aggregation and facilitates injection through micro-catheters. They are available in sizes ranging from $40 \mu \mathrm{m}$ to $1300 \mu \mathrm{m}$. Varying sizes of embolics have shown to correlate with vessel size and may allow for identification of a threshold for intratumoral penetration of embolics (Laurent et al., 2005).

\section{Gelfoam}

Gelfoam is a water-insoluble, porous, and pliable agent that resorbs completely within 4-6 weeks. Occlusion occurs after the gelfoam matrix facilitates thrombus formation. This agent can be used for embolization of highly vascular head and neck tumors for pre-operative resection to reduce blood loss. Both sponge and powder forms have been used in head and neck tumor embolization (Ruska et al., 1985). Gelfoam strips are rolled into a cylindrical shape and advanced through a catheter to the intended location. The Gelfoam powder is injected through a micro-catheter using a similar technique as particle embolism.

\section{LIQUID EMBOLIC AGENTS}

\section{N-butylcyanoacrylate}

$\mathrm{N}$-butyl cyanoacrylate is a liquid which polymerizes in the ionic and high $\mathrm{pH}$ environment in the body. A mixture of $\mathrm{n}-\mathrm{BCA}$ and ethiodol is prepared and micronized tantalum powder can be added to increase radiopacity for enhanced visualization. It is a good embolic agent for embolizing branches $0.5 \mathrm{~mm}$ or greater. It may not penetrate the tumor bed due to rapid polymerization upon contact with the blood and therefore an appropriate dilution is necessary for effectiveness. This will often demand a dilute preparation of $\mathrm{n}$-BCA, however care must be exercised in evaluating the flow dynamics because polymerization time will be extended which could lead to unintended embolization. Embolization of pedicles supplying the tumor can be performed by injecting $\mathrm{n}-\mathrm{BCA}$. If more than one feeder is present, each of the pedicles would need to be treated separately. Such an embolization approach may be useful in tumors with intratumoral shunting in which particle embolization may lead to transmission of particles to the venous system and resulting unintended distal embolization. Furthermore, if large pedicles are embolized, surgical resections should follow in the ensuing days. Delay in resection may lead to angiogenesis and growth of new vascular pathways to the tumoral bed. Embolization of vascular tumors with n-BCA has been associated with devascularization rates of up to $90-95 \%$ (Gupta et al., 2007).

\section{Ethyl vinyl alcohol polymer (ONYX)}

Onyx is a liquid embolic agent dissolved in dimethyl sulfoxide (DMSO). Upon contact with the blood, DMSO diffuses 
out and EVOH polymerizes. This polymer hardens on the outer surface first gradually polymerizing toward the core over several minutes. This property allows longer injections of this material. Using this technique, onyx can be used to embolize tumor bed. This liquid embolic agent can penetrate the tumor bed and can provide controlled embolization of the tumor vascular bed with good penetration.

\section{COILS}

Pushable and detachable coils can be used to embolize large $(>1.5 \mathrm{~mm})$ feeders to tumor beds. Coils afford control and are least likely to embolize distally into the draining veins. They can be an ideal embolization solution for tumors with intratumoral shunting. Detachable coils provide more control and can be adjusted during deployment. Coils with bio-active components can provide a nidus for thrombosis and eventual occlusion of the vessel. As with n-BCA embolization, coils provide proximal occlusion of the blood supply to the tumor bed without the embolization of the tumor vasculature itself. Therefore, surgical resection should follow coil embolization to minimize the development of collateral blood channels.

Various strategies have been employed to further improve thrombogenicity:

- Coatings: thrombin, gelatin, and polyurethane.

- Fibers: typically individual fibers or bundles of 5-100 fibers are used. Suitable fibers include synthetic materials Dacron, polyesters, polyamides, and natural fibers such as silk and cotton. The fibers are looped in a generally serpentine manner along the coil. The fibrous loops are affixed to (or looped through) the coil at spaced intervals along the coil.

\section{EMBOLIZATION TECHNIQUES}

Embolization approaches include:

- Transarterial

- direct puncture and

- combination of these both methods

\section{TRANSARTERIAL EMBOLIZATION}

This embolization technique includes coaxial super selective catheterization and infusion of embolic agent into the feeding arteries through a micro catheter. The objective is to infuse the embolic agent only into the feeders, thus sparing normal, uninvolved arteries. Optimal catheterization is as super selectively into the tumor feeder as possible. Reshaping of the microguide wire or microcatheter is usually necessary, depending on the course of the relevant vessels. High-resolution angiography with subtraction facilitates the procedure and road-mapping techniques are also necessary. If superselective catheterization is not available, or if multiple small branches supply the tumour, the normal branches distal to the tumour feeder can be catheterized and embolized with gelfoam strips or coils for protection, and then particles can be infused proximal to the feeders. Flow control techniques, using preferential flow or flow reversal, are used to make particles flow into the feeding branches to the tumours rather than into the normal branches when particles are infused proximal to the tumour branches. A repeat angiogram should be obtained immediately after embolization.

\section{DIRECT PUNCTURE EMBOLIZATION}

Local anaesthesia of the skin overlying the lesion should be obtained by infiltration of lignocaine $4 \%$ buffered with sodium bicarbonate $8.4 \%$ before doing the procedure. After selective transarterial angiography, the lesions are then punctured with an 18- to 20-G butterfly needle or i.v. cannula in the lesion and direct angiography is carried out with and without manual compression of the venous drainage in the region. In some cases, circumferential flow reduction around the lesion should be done. Then Cyanoacrylate-lipiodol mixture is injected as embolic agent. The concentration of the NBCA-iodized oil mixture is adjusted according to the flow rate as evaluated at angiography and using fluoroscopic road mapping during N-BCA injections which controlled the injection rate and volume. Repeated puncture and injections can be carried out when residual lesion is shown at postembolization angiography or in large lesions.

\section{COMPLICATIONS}

Complications from endovascular embolization include unintended occlusion from embolic material that can result from reflux of liquid embolic material and small particle size. Temporary balloon occlusion can be used as a technique to control placement of embolic material. Particle size must be chosen appropriately to allow for controlled delivery and catheter placement must be distal to origin of vessels to be preserved. Cranial nerve palsies may result from ischemia to the vasa nervorum and can be reduced with awareness of potentially harmful anastomoses (Figure 2).Major complications include blindness from ophthalmic artery occlusion and ECA to ICA anastomosis emboli resulting in stroke. Minor adverse events include facial pain resulting from ischemia and can be managed with appropriate analgesia. Bradycardia from a trigeminocardiac reflex has been reported in association with manipulation of the external carotid artery branches, which likely involves trigeminal nerve afferent tracts to the vagus nerve ( $\mathrm{Lv}$ et al., 2007). This has also been reported with internal maxillary artery embolization. Atropine should be available for severe bradycardia

\section{CONCLUSION}

Endovascular embolization for head and neck tumors can provide an effective adjunctive therapy to resection. A thorough understanding of the angioarchitecture of head and neck tumors and neurovascular anastomoses can play an important role in diagnosis and treatment planning. Novel techniques and embolic agents will continue to advance endovascular treatment, and emerging approaches including intra-arterial chemotherapy may expand the endovascular options for cancer therapy.

\section{REFERENCES}

1. Gampper TJ, Morgan RF. Vascular anomalies: hemangiomas. Plast Reconstr Surg 2002; 110(2):572585 .

2. Burns AJ, Navarro JA, Cooner RD. Classification 
of vascular anomalies and the comprehensive treatment of hemangiomas. Plast Reconstr Surg 2009; 124(5):69e-81e.

3. Smith TP. Embolization in the external carotid artery. J Vasc Interv Radiol 2006; 17(1):1897-912.

4. Jenson ME. Endovascular and percutaneous therapy for extra cranial tumors. In: Marks MP, Do HM, editors. Endovascular and percutaneous therapy of the brain and spine. Philadelphia: Lippincott Williams \& Wilkins. 2002. p. 361-88.

5. Marc A. Lazzaro, et al. Endovascular embolization of head and neck tumors. Frontiers in Neurology. 2011; 2(4):64.

6. Gemmete et al. Embolization of Vascular Tumors of the Head and Neck. Neuroimag Clin N Am 2009; 19(6):181-198.

7. Damascelli, B., et al. Feasibility and efficacy of percutaneous transcatheter intra-arterial chemotherapy with paclitaxel in albumin nano particles for advanced squamous cell carcinoma of the oral cavity, oropharynx, and hypopharynx. J. Vasc.Interv.Radiol. 2007;18(1):1395-1403.

8. Gupta et al. Preoperative embolization of hyper vascular head and neck tumours. Australasian Radiology 2007;51:446-452.

9. Tony P. Smith. Embolization in the External Carotid Artery. J Vasc Interv Radiol 2006; 17(5):1897-1913.

10. Sepulveda et al. Vascular Tumors. Semin Plast Surg 2014;28(3):49-57

11. Roberson et al. Therapeutic Embolization of Juvenile Angiofibroma. AJR 1979;133(6):657-663.

12. Quadros et al. Preoperative Embolization of a Cervicodorsal Paraganglioma by Direct Percutaneous Injection of Onyx and Endovascular Delivery of Particles. AJNR Am J Neuroradiol 2006;27(4):190709.

13. Abud et al. Intratumoral Injection of Cyanoacrylate Glue in Head and Neck Paragangliomas. AJNR Am J Neuroradiol 2004;25(2):1457-1462.

14. Yutaka Kai et al. Appropriate Interval between Embolization and Surgery in Patients with Meningioma. AJNR Am J Neuroradiol 2002;23(5):139-142

Source of Support: Nil; Conflict of Interest: None

Submitted: 02-12-2019; Accepted: 30-12-2019; Published online: 28-01-2020 